\title{
Ghost stochastic resonance in vertical-cavity surface-emitting lasers: Experiment and theory
}

\author{
Guy Van der Sande,* Guy Verschaffelt, and Jan Danckaert \\ Vrije Universiteit Brussel, Department of Applied Physics and Photonics (TW-TONA), Pleinlaan 2, 1050 Brussels, Belgium \\ Claudio R. Mirasso \\ Universitat de les Illes Balears, Departament de Fisica, E-07122 Palma de Mallorca, Spain \\ (Received 5 July 2004; revised manuscript received 13 May 2005; published 18 July 2005)
}

\begin{abstract}
We study the polarization response of a vertical-cavity surface-emitting laser, driven simultaneously by noise and two (or more) weak periodic signals. In the bistable regime, we observe experimentally the occurrence of stochastic resonance at a frequency that is absent in the input driving signal. The presence of this so-called ghost resonance is confirmed theoretically.
\end{abstract}

DOI: 10.1103/PhysRevE.72.016113

PACS number(s): 05.40.-a, 42.65.Sf, 42.55.Px

\section{INTRODUCTION}

Subharmonic stochastic synchronization and resonance, amiably referred to as ghost resonance, were first studied in the framework of the auditory system $[1,2]$. This study originated from the search for the neuronal process by which the brain fuses all the sound's frequency components into a perceived pitch that is not present in the original sound wave. This ghost resonance (GR) is a form of stochastic resonance (SR) whereby a frequency absent in the input shows up in the system response. This is a type of phenomenon which of course is not possible within the framework of linear signal processing. Until now, GR has been observed theoretically in an excitable system $[1,2]$ and experimentally in a laser system [3] and in a monostable Schmitt-Trigger electronic circuit [4]. Here, we demonstrate the occurrence of GR in a bistable system.

SR has been shown both experimentally $[5,6]$ and theoretically [7] in polarization-switching (PS) vertical-cavity surface-emitting lasers (VCSEL's). VCSEL's operating in the fundamental transverse mode usually emit light in one of two specific orthogonal linear polarizations states. In some devices the emitted polarization changes at a specific switching current. Around this switching current a small bistable region, where spontaneous mode hopping can be observed between the two modes, can exist. When the current is modulated in this region, SR is observed [5,6]. In [7], SR in VCSEL's was studied on the basis of a two-polarizationmode rate equation model. By using a multiple-time-scale analysis of these stochastic rate equations, the model is reduced to one stochastic differential equation. In that way, semianalytical results are far more easy to attain.

In this paper we study the response of a VCSEL subject to weak periodic input currents and a Gaussian white noise simultaneously. We will show that under appropriate conditions we can observe the presence of GR, both experimentally and theoretically, around the PS point of the VCSEL.

*Electronic address: guy.van.der.sande@vub.ac.be

\section{EXPERIMENTS}

\section{A. Measurements}

The VCSEL's used in the experiments are oxide-confined devices with a circular surface relief intended to increase the single-mode regime and emitting at $970 \mathrm{~nm}$ [8]. These devices were fabricated by the Optoelectronics Department of the University of Ulm. In our setup, the light emitted by the VCSEL is collimated with a lens and sent through a linear polarizer to select one of the two polarization modes (PM's). The polarization-resolved intensity impinges on a 2-GHz-bandwidth detector whose output signal is visualized on an oscilloscope. All optical elements are slightly misaligned to prevent optical feedback, which could cause extra instability in the VCSEL. The bias current generated by a stabilized current source and the modulation signal from a function generator are combined in a bias $T$ and subsequently sent through the VCSEL. We use a Gaussian white electrical noise source with a bandwidth of $400 \mathrm{MHz}$. The temperature of the VCSEL package is actively stabilized up to a few $\mathrm{mK}$. In all of our experiments discussed here we use a fixed temperature of $22{ }^{\circ} \mathrm{C}$. The overall amplitude response (from signal generator to laser current) was checked as being linear in the modulation amplitude and frequency regime that is studied here.

The PI curve of the studied VCSEL is shown in Fig. 1. The VCSEL used in the experiments has a threshold current $I_{t h}$ of about $0.84 \mathrm{~mA}$ and a switching current $I_{s w}$ of $1.38 \mathrm{~mA}$. Usually, random mode hopping between the two orthogonally polarized fundamental modes occurs within a bistable region around the switching current. However, in our case the mode hopping can only be observed close to the switching current, because the bistable region is too wide. From comparison with theory we estimate the bistable region to lie within $I_{L}<I<I_{H}$ with $I_{L}=1.14 \mathrm{~mA}$ and $I_{H}=3.05 \mathrm{~mA}$.

The VCSEL is modulated by the following current signal:

$$
I=I_{b}+\frac{I_{m}}{2}\left[\sin \left(2 \pi f_{1} t\right)+\sin \left(2 \pi f_{2} t\right)\right]
$$

where

$$
f_{1}=k f_{0}+\Delta f,
$$




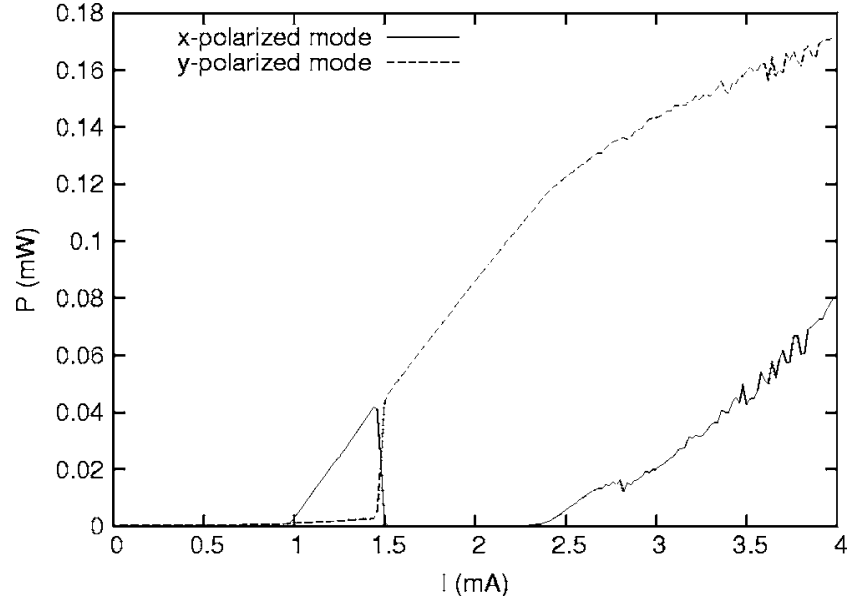

FIG. 1. Polarization-resolved PI scan of the VCSEL used in the experiments. This VCSEL has a switching current $I_{s w}$ of $1.38 \mathrm{~mA}$. The threshold current $I_{\text {th }}$ is about $0.84 \mathrm{~mA}$.

$$
f_{2}=(k+1) f_{0}+\Delta f
$$

With this type of signal the ghost resonance is expected at frequency [1]

$$
f_{r}=f_{0}+\frac{\Delta f}{k+1 / 2} .
$$

This resonance can be better understood, when assuming that the nonlinear system acts as a thresholding device and merely detects the position of the highest peaks produced by constructive interference of the two sinusoidal signals with equal amplitudes. In that case, Eq. (4) corresponds to the time interval between two prominent peaks. Although ghost resonance can be observed for a large combination of sine functions, we choose to work with $k=2$ and only two input frequencies. To ensure that only one resonance frequency $f_{r}$ exists, we start with $\Delta f=0$. Remark that for $\Delta f=0$, the resonance will occur, as expected, at the subharmonic frequency $f_{0}$ that is absent in the injected current signal. When $\Delta f \neq 0$, the resonance does not occur simply at the frequency difference $f_{2}-f_{1}$, but at a slightly shifted frequency as follows from Eq. (4).

To set $I_{b}$, we have considered two situations: $I_{b}$ lower than the Maxwell point and $I_{b}$ higher than the Maxwell point. The Maxwell point is the value of the current at which the average residence times, due to the noise-induced mode hopping, in both modes are equal. In both cases, we have observed ghost resonance. The measurements with a bias current below the Maxwell point are quite time consuming as the resonance occurs at a time scale close to seconds, while in the other case the involved time scale is closer to microseconds. Taking into account that we have to measure the VCSEL's response during several thousand modulation cycles in order to get statistically relevant data, it is clear that the second case leads to easier and less time-consuming measurements. That is why the focus of this paper is on the case with the bias current above the Maxwell point.
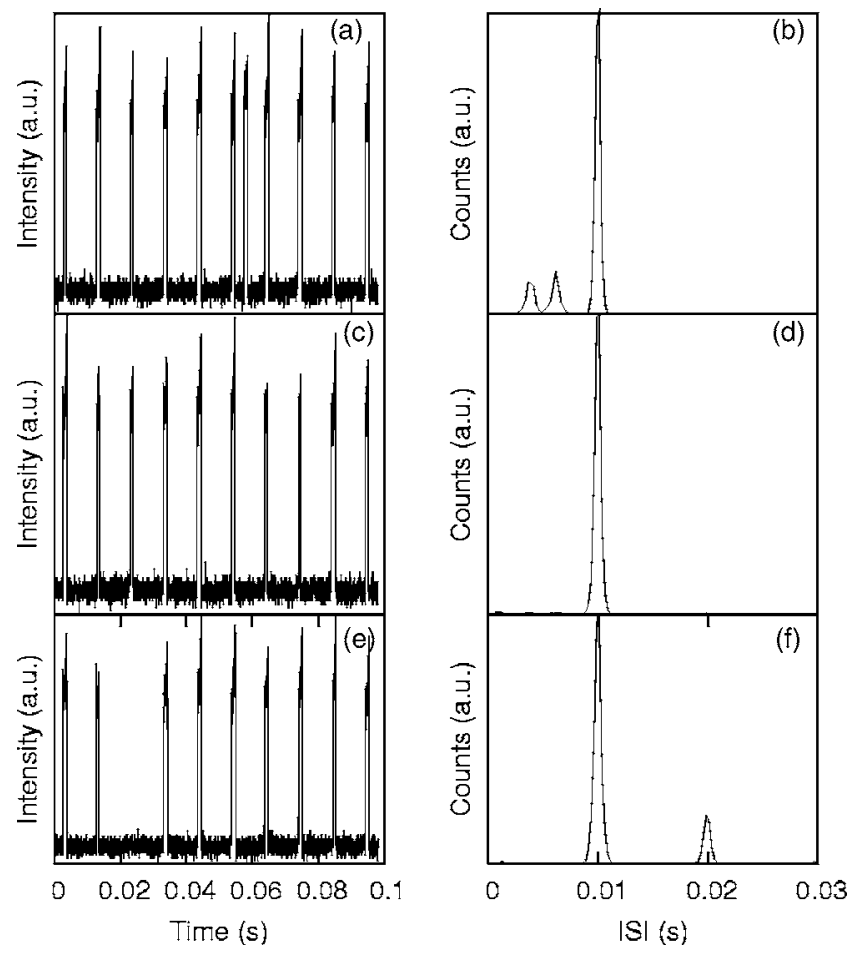

FIG. 2. Time traces of the intensity in the polarization mode of interest and their corresponding ISI histograms for a fixed $I_{b}$ $=1.54 \mathrm{~mA}$ and $f_{0}=100 \mathrm{~Hz}(k=2, \Delta f=0)$ and different modulation amplitudes: $I_{m}=0.19 \mathrm{~mA}$ in panels (e) and (f), $I_{m}=0.22 \mathrm{~mA}$ in panels (c) and (d), and $I_{m}=0.26 \mathrm{~mA}$ in panels (a) and (b).

Typical time traces for a fixed $I_{b}$ and $f_{0}$ and for different modulation amplitudes can be found in Fig. 2. In Fig. 2(c) the system is clearly very close to its resonance point. It exhibits a spiked response with a repetition rate equal to $f_{0}$. Figures 2(a) and 2(e) are the two typical out-of-resonance cases. In Fig. 2(e) some spikes are missed, which indicates that the system is not always able to respond to the input signal and some hops are missed. In Fig. 2(a) intermediate spikes sometimes occur at a rate equal to the frequency of one of the two sines of the input signal. Due to the asymmetry in residence time distributions for the two polarization modes, the ghost resonance can be better characterized by measuring the histograms of the interspike intervals (ISI's) $[9,10]$. The interspike interval is defined as the time between the rising edges of two consecutive pulses or spikes. In what follows we will investigate ghost resonance, varying the amplitude, the frequency, and the noise level of the input signal.

\section{B. Results}

\section{Amplitude resonance}

We have measured the ISI distribution for a fixed frequency $f_{0}=100 \mathrm{~Hz}, k=2, \Delta f=0$, and different modulation strengths $I_{m}$ ranging from $0.17 \mathrm{~mA}$ to $0.30 \mathrm{~mA}$. In Fig. 3 the results are summarized. In Figs. 3(a)-3(c) the ISI histograms are plotted considering different modulation strengths $\left(I_{m}\right.$ $=0.19 \mathrm{~mA}, I_{m}=0.22 \mathrm{~mA}$, and $I_{m}=0.26 \mathrm{~mA}$, respectively). Figure 3(a) corresponds to a modulation strength $I_{m}$ below 

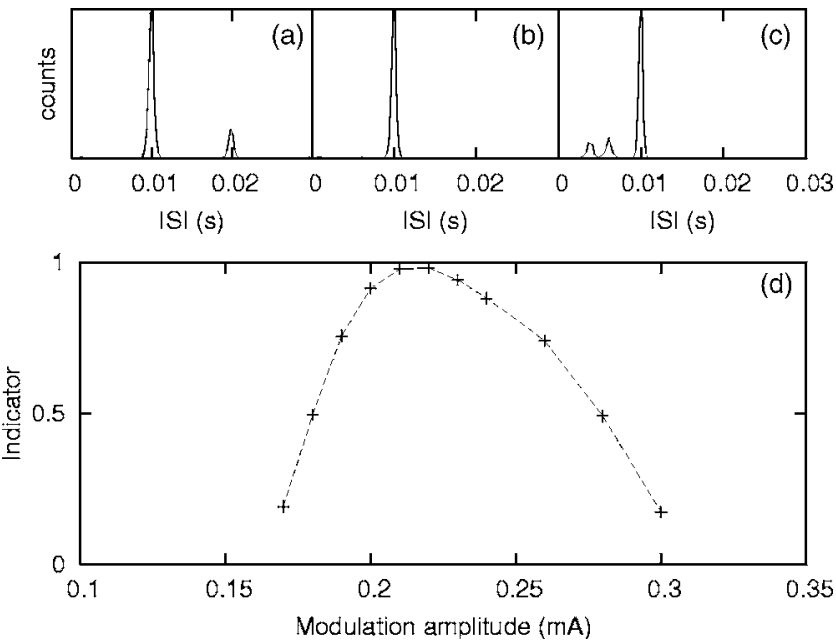

FIG. 3. ISI histograms when the VCSEL is driven by a signal with $I_{b}=1.54 \mathrm{~mA}, f_{0}=100 \mathrm{~Hz}, \Delta f=0$, and $k=2$. The modulation

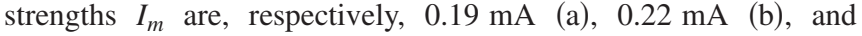
$0.26 \mathrm{~mA}$ (c). (d) The ghost resonance indicator is plotted as a function of modulation strength.

the resonance where some spikes are occasionally missed [see the time trace in Fig. 2(a)]. In Fig. 3(b) the system is in resonance and the ISI histogram shows a strong peak corresponding to the missing fundamental. This case corresponds to the time trace in Fig. 2(b). For higher modulation strengths [Fig. 3(c)], we see that spikes sometimes occur at time scales related to the frequency present in the signal as in Fig. 2(c). To quantify the resonant behavior we use the indicator proposed in Sec. III [5,11-15]:

$$
I_{T}=\frac{\int_{T-T / 4}^{T+T / 4} P_{m}(t) d t}{\int_{0}^{\infty} P_{m}(t) d t},
$$

where the ISI histogram $P_{m}$ is integrated around the peak present in the vicinity of the period $T=1 / f_{0}$. The result is plotted as a function of the modulation strength in Fig. 3(d). This indicator reaches a maximum in the vicinity of $I_{m}$ $=0.22 \mathrm{~mA}$. At that point GR is reached.

\section{Frequency resonance}

Starting from the resonance point of the previous section $\left(I_{b}=1.54 \mathrm{~mA}, I_{m}=0.22 \mathrm{~mA}, \Delta f=0\right.$, and $\left.k=2\right)$, we have made a similar study, varying the fundamental frequency $f_{0}$ from $2 \mathrm{~Hz}$ to $4 \mathrm{kHz}$. The results are summarized in Fig. 4 in which we again plot the indicator $I_{T}$ as obtained from the ISI histograms, now for different $f_{0}$. We see that for low frequencies we are in the case of occasional unwanted spikes [Fig. 4(a)], while the higher frequencies [Fig. 4(c)] lead to a scenario of occasionally missed spikes. We have plotted the indicator given by Eq. (5) as a function of $f_{0}$ in Fig. 4(d). One can observe a resonance behavior in a wide region around $100 \mathrm{~Hz}$.
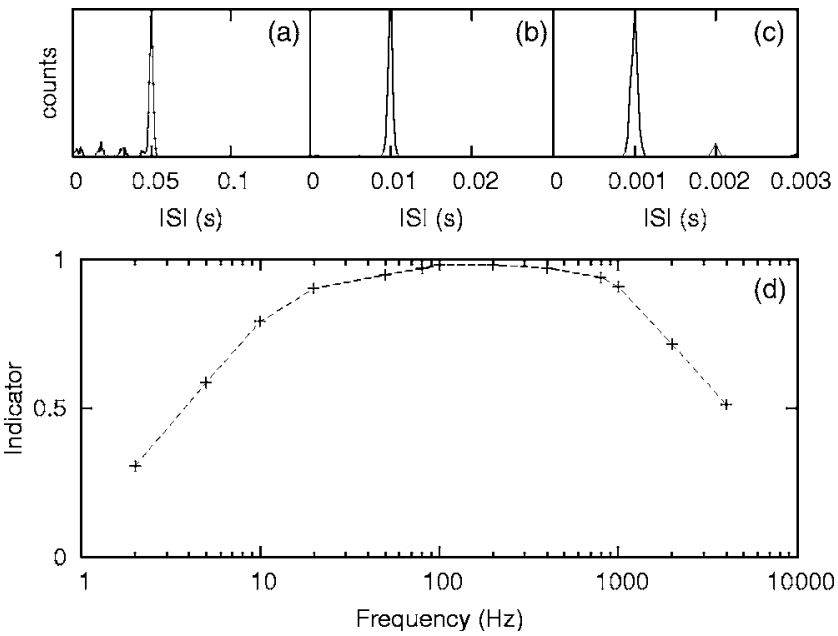

FIG. 4. ISI histograms when the VCSEL is driven by a signal with $I_{b}=1.54 \mathrm{~mA}, I_{m}=0.22 \mathrm{~mA}, \Delta f=0$, and $k=2$. The fundamental frequencies $f_{0}$ are, respectively, $20 \mathrm{~Hz}$ (a), $100 \mathrm{~Hz}$ (b), and $1000 \mathrm{~Hz}$ (c). (d) The ghost resonance indicator is plotted as a function of the fundamental frequency.

\section{Noise resonance}

Finally, we have checked the dependence of GR on the noise level of the input signal. Again, we can see a resonancelike behavior in Fig. 5(d). In Figs. 5(a)-5(c) the corresponding ISI histograms are plotted. The noise level indicated in Fig. 5 corresponds to noise added on the input current signal. The noise can be considered white up to frequencies of about $400 \mathrm{MHz}$, which is much larger than all the time scales involved in the experiment. The same scenario as in [1] has been reproduced. For low noise levels, the VCSEL sends out spikes at the wanted repetition rate, but occasionally misses one or more of them, while at high noise levels the frequencies present in the input signal are excited as well and the VCSEL emits spikes at undesired repetition
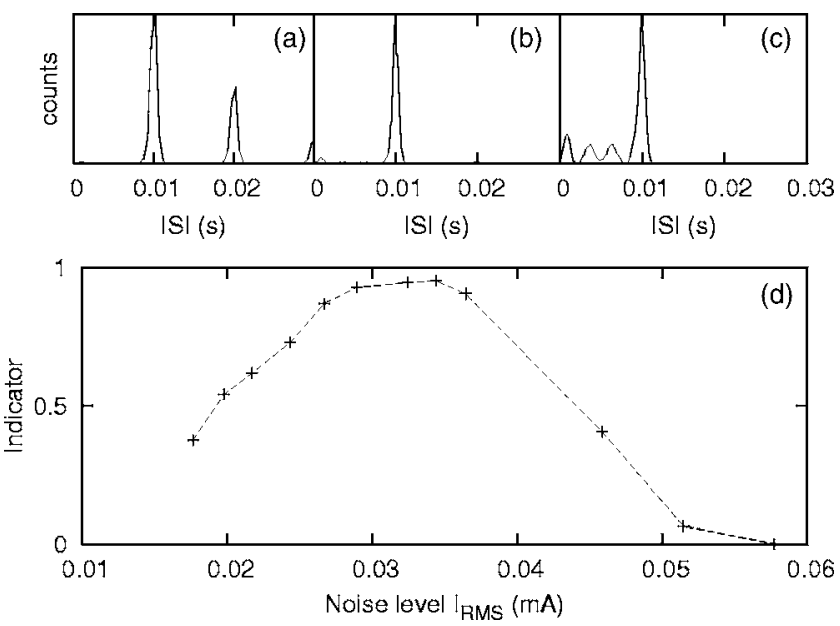

FIG. 5. ISI histograms when the VCSEL is driven by a signal with $I_{b}=1.54 \mathrm{~mA}, f_{0}=100 \mathrm{~Hz}, I_{m}=0.18 \mathrm{~mA}, \Delta f=0$, and $k=2$. The noise levels are, respectively, $I_{r m s}=0.020 \mathrm{~mA}$ (a), $0.032 \mathrm{~mA}$ (b), and $0.046 \mathrm{~mA}$ (c). (d) The ghost resonance indicator is plotted as a function of noise level. 


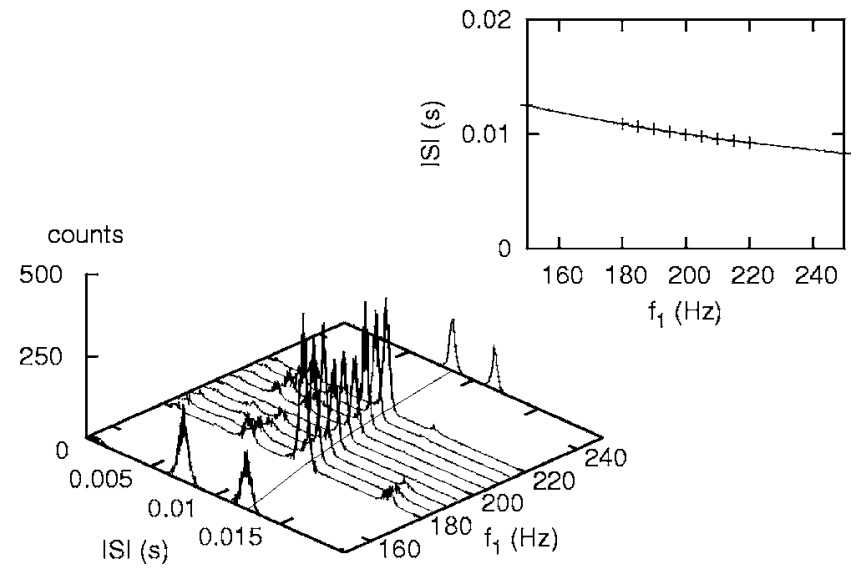

FIG. 6. Several ISI histograms plotted for different $f_{1}$ (and $f_{2}$ $\left.=f_{1}+f_{0}\right)$. The rest of the parameters are $I_{b}=1.54 \mathrm{~mA}$ and $I_{m}$ $=0.22 \mathrm{~mA}$. The solid line in the (ISI- $\left.f_{1}\right)$ plane is the theoretical predicted resonance repetition rate. In the inset the measured resonance frequency (points) and the theoretical prediction (line) are compared.

rates. Again, one can observe a resonant behavior around an "optimum" noise level of about $I_{r m s}=0.032 \mathrm{~mA}$.

The observation of a resonance both in frequency and noise level indicates that the external modulation time scale matches the internal stochastic time scales of the system $[5,11,16]$. The stochastic time scales involved in this problems are the residence times of the polarization modes within the bistable region, as will be discussed in Sec. III. The timescale matching condition for this problem is not straightforward and, as commented on in [10], reduces to $\left\langle T_{+}\right\rangle+\left\langle T_{-}\right\rangle$ $=1 / f_{r}$, where $\left\langle T_{ \pm}\right\rangle$are the mean residence times of the modes.

\section{Inharmonic resonance}

In this section, we concentrate on inharmonic signals and ghost resonance. In this case $\Delta f$ is no longer zero and the resonance frequency $f_{r}$ does not correspond to the missing fundamental $f_{0}$. To study this type of signal we start from the frequencies $f_{1}=200 \mathrm{~Hz}$ and $f_{2}=300 \mathrm{~Hz}$ and we shift these two frequencies by the same amount. The bias current $I_{b}$ and the modulation strength $I_{m}$ are fixed at $1.54 \mathrm{~mA}$ and $0.22 \mathrm{~mA}$, respectively. In Fig. 6 we plot the measured ISI histograms for several values of $f_{1}$. The line plotted in the ISI vs $f_{1}$ plane is the resonance repetition rate as a function of $f_{1}$ given by Eq. (4) for the values of $f_{0}=100 \mathrm{~Hz}$ and $k=2$. At the edges of the figure, one can see the appearance of a second resonance repetition rate [because at those points two sets of $(\Delta f, k)$ can describe the signal]. One can also see that the energy is evenly distributed between the two resonance repetition rates. In the inset the maxima of the resonance ISI and the theoretical prediction given by Eq. (4) are compared. We find a perfect match.

\section{TWO-MODE STOCHASTIC RATE EQUATION MODEL}

We model the polarization behavior of VCSEL's using a two-mode rate equation description where we study the evo- lution of charge carriers and the photon densities of the two polarization modes. This approach has proven to be successful in explaining the stationary polarization characteristics [17], the mode hopping phenomenon [18], and also the phenomenon of stochastic resonance in VCSEL's [7]. In reduced form, these equations read

$$
\begin{gathered}
\frac{d p_{x}}{d t}=p_{x}\left(n-\varepsilon_{s x} p_{x}-\varepsilon_{x y} p_{y}\right)+\frac{1}{2} R_{s p}+\widetilde{F}_{x}, \\
\frac{d p_{y}}{d t}=p_{y}\left[n+G(J)-\varepsilon_{s y} p_{y}-\varepsilon_{y x} p_{x}\right]+\frac{1}{2} R_{s p}+\widetilde{F}_{y}, \\
\frac{d n}{d t}=\frac{J-p_{x}-p_{y}}{\rho}-p_{x}\left(n-\varepsilon_{s x} p_{x}-\varepsilon_{x y} p_{y}\right) \\
-p_{y}\left(n-\varepsilon_{s y} p_{y}-\varepsilon_{y x} p_{x}\right)+\tilde{F}_{n},
\end{gathered}
$$

with

$$
\begin{gathered}
\left\langle\widetilde{F}_{x, y}(t) \tilde{F}_{x, y}(s)\right\rangle=2 R_{s p} p_{x, y} \delta(t-s), \\
\left\langle\widetilde{F}_{x}(t) \widetilde{F}_{y}(s)\right\rangle=0 .
\end{gathered}
$$

The equations need to be interpreted in the Stratonovich sense. Here, the time has been rescaled to the carrier lifetime and $\rho$ is the ratio of the photon and carrier lifetimes ( $\approx 1 \mathrm{~ns}$ ), which is typically $10^{-3}$. The photon densities $p_{x, y}$ are reduced such that $p_{x(y)}=J$ if the $x(y)$ mode is lasing, respectively, $J$ being the reduced current. $n$ is the deviation from the value of the carrier density at threshold. The parameters $\varepsilon_{s x, s y, x y, y x}$ and $G(J)$ are gain saturation coefficients and current-dependent linear gain difference, whereas $R_{s p}$ is the noise strength.

Experimentally, it is only possible to increase the noise level on the injection current. From our experiments we know that the effect of increasing the noise on the current is to decrease the stochastic time scales. Theoretically, we are able to do the same by increasing the spontaneous emission noise. So, at least at a qualitative level, both types of noise have the same effect on the stochastic residence time scales. We will choose to neglect the noise in our model on the current, because in this case we will be able to obtain semianalytical results. If we were to put the noise on the current in the model, we can only obtain results by numerically integrating the stochastic differential equations (6)-(8), which is very time consuming and does not lead to more physical insight into GR. We have therefore limited our work to the presented analytical approach. So, neglecting carrier noise and considering time scales slower than the relaxation oscillations, Eqs. (6) -(8) can be further reduced to a single dynamical equation [7] of the form

$$
\frac{d p_{y}}{d t}=C\left(p_{y}\right)+\tilde{F}\left(p_{y}\right),
$$

with a deterministic drift term 


$$
C\left(p_{y}\right)=p_{y}\left(J-p_{y}\right)\left(\frac{2 \delta}{J} p_{y}-\delta+\frac{G}{J}\right)+\frac{R_{s p}}{2 J}\left(J-2 p_{y}\right)
$$

and a stochastic term

$$
\widetilde{F}\left(p_{y}\right)=\widetilde{F}_{y}-\frac{\tilde{F}_{x}+\tilde{F}_{y}}{J} p_{y},
$$

where $\delta$ is defined as

$$
\delta=\varepsilon_{x y}-\varepsilon_{s x}=\varepsilon_{y x}-\varepsilon_{s y}
$$

and where we have taken the symmetry of a VCSEL into account. The equation for the $x$ mode can be found by interchanging $x$ and $y$ and by changing the sign of $G$. This reduction is only valid on time scales slower than the relaxation oscillations. The validity of the reduction from Eqs. (6)-(8) to Eq. (11) was proven in Ref. [18]. As the modulation frequencies considered here will never exceed $10 \mathrm{MHz}$, we can safely adopt this approach.

In this work, we assume that $G$ is positive at threshold and linearly drops with the injected current. At a certain current $J_{s}$, the switching current, it changes sign. So the current dependence of the gain difference is expressed as

$$
G(J)=g\left(1-\frac{J}{J_{s}}\right)
$$

In that case, keeping in mind that $\delta>0$, the $y$-polarized mode will start lasing at threshold, but the device will transit through a bistable region (around $J=J_{s}$ ) and eventually switch to the $x$-polarized mode.

In the bistable region we can expect stochastic mode hopping to occur [18]. From now on, we will call $p_{y} \simeq J$ mode the "+" state and $p_{y} \simeq 0$ the "-" state. The residence times, being the mean times the system dwells in a certain state before switching to the other state at (a fixed current), of these two states are given by [18]

$$
t_{ \pm}=\frac{2 J \pi \delta}{J^{2} \delta^{2}-G^{2}} \operatorname{erf}\left(\frac{G \pm J \delta}{2 \sqrt{R_{s p} \delta}}\right) \operatorname{erfi}\left(\frac{G \pm J \delta}{2 \sqrt{R_{s p}} \delta}\right) .
$$

These residence times depend strongly on current, not only directly, but also through the current dependent gain $G(J)$. The scaling of the residence times $t_{ \pm}$with $J$ is shown in Fig. 7. The parameters used in Fig. 7 are extracted from a quantitative comparison between the theoretical predictions and measurements in the mode hopping regime for the VCSEL used in the experiment. These parameters will be used in the remainder of this paper. In Fig. 8 we have plotted three realizations of $p_{y}(t)$ for different values of $R_{s p}$ as obtained numerically from Eq. (11). A similar behavior as in the experimental time traces can be observed.

To investigate the ghost resonance, we need to look at the statistical properties of the inter spike intervals. These can of course be obtained from time traces as in Fig. 8 (see, e.g., the numerically obtained ISI distribution in Fig. 9). Generating a statistically relevant amount of events from a numerical integration of Eq. (11) is quite time consuming, because Eq. (11) is governed by two very different time scales. The first is the period of the pulses (which is, e.g., $10 \mathrm{~ms}$ ). The second

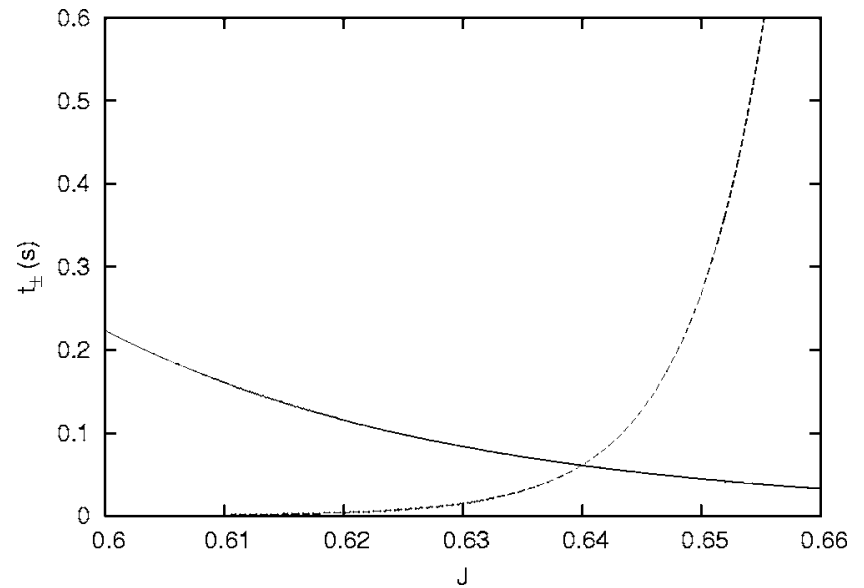

FIG. 7. The average residence times $t_{+}$(solid lines) and $t_{-}$ (dashed lines) as a function of the injected current $J(\delta=3.9, g$ $=3.8, J_{s}=0.64, R_{s p}=0.021$ ).

time scale is the switching time (the rising edge and falling edge of each spike, which is around $1 \mathrm{~ns}$ ). Therefore, we have opted for a semianalytical approach. Also, the parameter space in a ghost resonance problem can be quite big (bias current, modulation amplitude, frequency, etc.). That is why an automated semianalytical approach can be a more practical tool compared to experimental trial-and-error based measurements.

The ISI distributions can be retrieved from the statistics of the residence times. When the injected current is kept constant, the residence times are exponentially distributed, but when the current is modulated at a slow time scale, the residence time distribution becomes

$$
P_{m \pm}\left(t \mid t_{0}\right)=\frac{1}{t_{ \pm}(t)} \exp \left(-\int_{t_{0}}^{t_{0}+t} \frac{d u}{t_{ \pm}(u)}\right) .
$$

This distribution function represents the probability that the VCSEL switches at a time $t_{0}+t$ when at $t_{0}$ the VCSEL is in

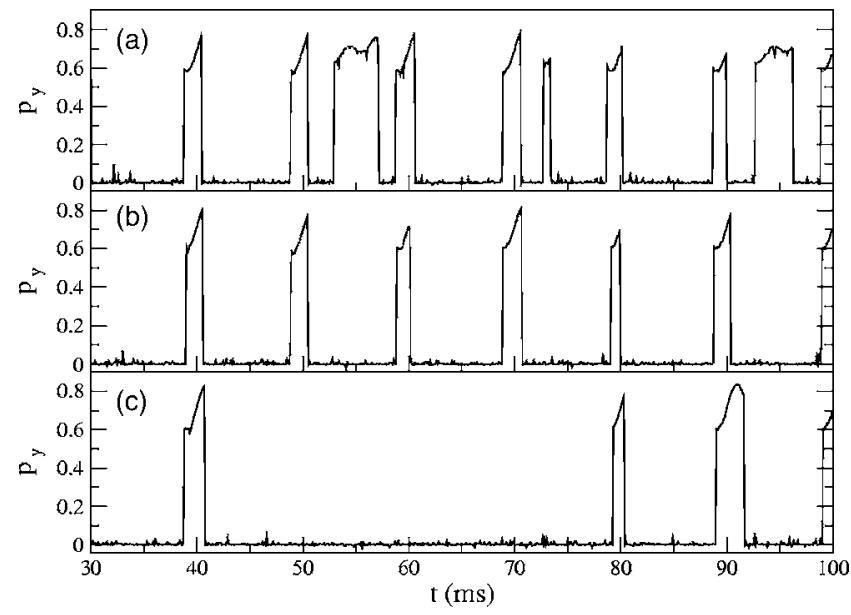

FIG. 8. $p_{y}$ as obtained numerically from Eq. (11) for different noise strengths. (a) $R_{s p}=0.023$, (b) $R_{s p}=0.021$, and (c) $R_{s p}=0.019$. The modulation signal is defined as $J=J_{b}+J_{m} / 2\left(\sin 4 \pi f_{0} t\right.$ $\left.+\sin 6 \pi f_{0} t\right) \quad\left(\delta=3.9, \quad g=3.8, \quad J_{s}=0.64, \quad J_{b}=0.70, \quad J_{m}=0.12, \quad f_{0}\right.$ $=100 \mathrm{~Hz}$ ). 


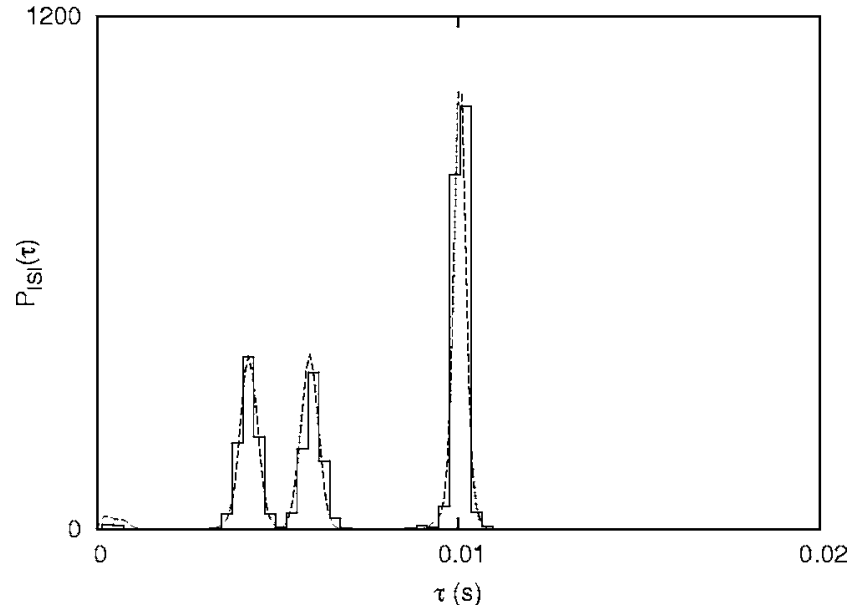

FIG. 9. Comparison between the ISI distribution obtained by numerical integration of Eq. (11) (solid line) and the ISI distribution as predicted by the semianalytical theory presented in the Appendix (dashed). Parameters as in Fig. 8(b).

the \pm mode. In practice, $t_{0}$ will be chosen at the point where a switch occurs to the \pm mode. From the knowledge of the residence time distributions $P_{m \pm}\left(t \mid t_{0}\right)$, we can find the probability density function of the interspike intervals. The spikes in the experiment represent a jump from the - mode to the + mode and back. The time interval between the rising edges of consecutive peaks is called the interspike interval. The conditional ISI distribution, when the last spike occurred at $\tau_{T}$, is then given by

$$
P_{I S I}\left(\tau \mid \tau_{T}\right)=\int_{0}^{\tau} P_{m+}\left(s \mid \tau_{T}\right) P_{m-}\left(\tau-s \mid \tau_{T}+s\right) d s .
$$

In Fig. 10, one realization of an interspike interval is

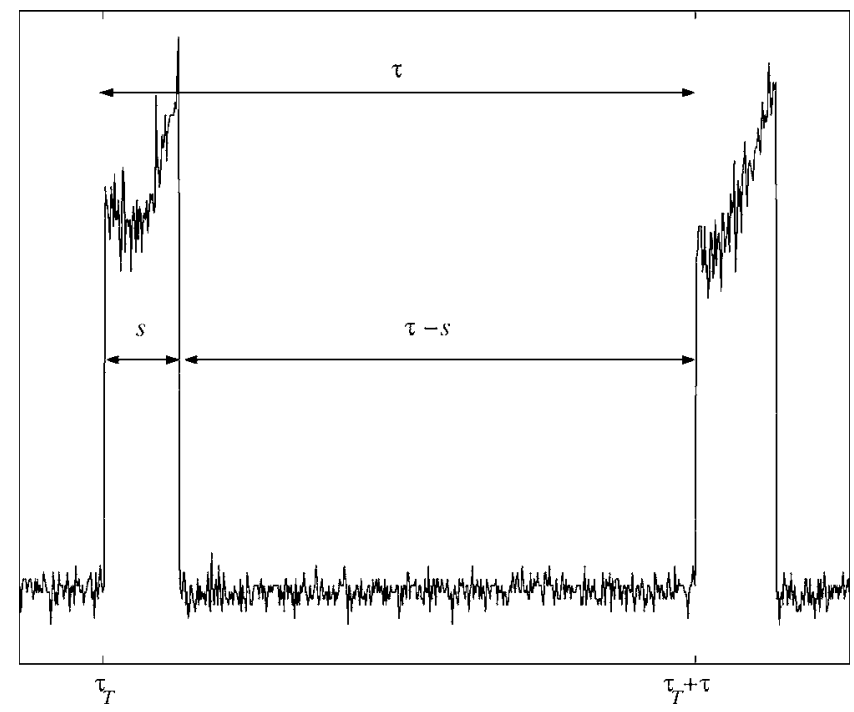

FIG. 10. A typical realization of an interspike interval. At $\tau_{T}$, the VCSEL switches to the + mode, and after a time $s$ drops back to the - mode. The VCSEL is lasing in the - mode at $\tau_{T}+s$ and switches to the + mode at a time $\tau-s$ later, completing the interspike interval. shown. At $\tau_{T}$, the VCSEL switches to the + mode, and after a time $s$ it drops back to the - mode. The probability for this to occur is $P_{m+}\left(s \mid \tau_{T}\right)$. The VCSEL is lasing in the - mode at $\tau_{T}+s$ and switches to the + mode at a time $\tau-s$ later, completing the interspike interval. The probability for this event to happen equals $P_{m-}\left(\tau-s \mid \tau_{T}+s\right)$. The multiplication of both probabilities will give the probability for this realization of an interspike interval of $\tau$ to occur. Integration over all realizations (integration over $s$ ) gives the conditional ISI distribution $P_{I S I}\left(\tau \mid \tau_{T}\right)$.

It should be noted that $P_{I S I}\left(\tau \mid \tau_{T}\right)$ is still not the ISI distribution that is experimentally recorded. Indeed, Eq. (18) depends on the spike time $\tau_{T}$, the time where the first rising edge occurs. In this discussion, we will temporarily limit ourselves to a periodic modulation with period $T$ (i.e., $\Delta f$ $=0)$. Then, $\tau_{T}$ can be limited to within one modulation period $\left(0 \leqslant \tau_{T}<T\right)$. In this sense, $\tau_{T}$ connects an event (a rising edge) to a point within one period of the modulation signal ( $\tau_{T}$ can be considered as the "phase" of the periodic signal). To get the measured $\left(\tau_{T}\right.$-independent) ISI distribution, we need to include in our description the spike time distribution $P\left(\tau_{T}\right)$, being the probability density of a spike starting at $\tau_{T}$. The $\tau_{T}$-independent ISI distribution $P_{I S I}(\tau)$ is then given by

$$
P_{I S I}(\tau)=\int_{0}^{T} P_{I S I}\left(\tau \mid \tau_{T}\right) P\left(\tau_{T}\right) d \tau_{T} .
$$

Even if one could solve Eq. (18) analytically, it would be hard (if not impossible) to deduce the distribution $P_{I S I}(\tau)$ or $P\left(\tau_{T}\right)$ analytically. In the Appendix, we show that from the analytical formulation of the residence times in Eq. (16) it is possible to calculate $P\left(\tau_{T}\right)$, and consequently $P_{I S I}(\tau)$, semianalytically.

We use Eq. (19) and the approximate way of determining the spike time distribution $P\left(\tau_{T}\right)$ as described in the Appendix to confirm the presence of ghost resonance theoretically. We concentrate on the noise-induced ghost resonance and the frequency resonance. As indicator to identify the presence of GR, we use

$$
I_{T}=\int_{T-1 / 4 T}^{T+1 / 4 T} P_{I S I}(\tau) d \tau .
$$

This indicator is based on the indicators defined in [5,11-15] with the difference that the background distribution (i.e., the distribution in the absence of current modulation) is neglected and not subtracted from Eq. (20). We can safely neglect the contributions from the background, as the probability level of the background is much lower than that of the spiked ISI distributions. To quantify the out-of-resonance behavior, we propose the indicators

$$
\begin{gathered}
I_{L}=\int_{0}^{T-1 / 4 T} P_{I S I}(\tau) d \tau, \\
I_{H}=\int_{T+1 / 4 T}^{+\infty} P_{I S I}(\tau) d \tau .
\end{gathered}
$$



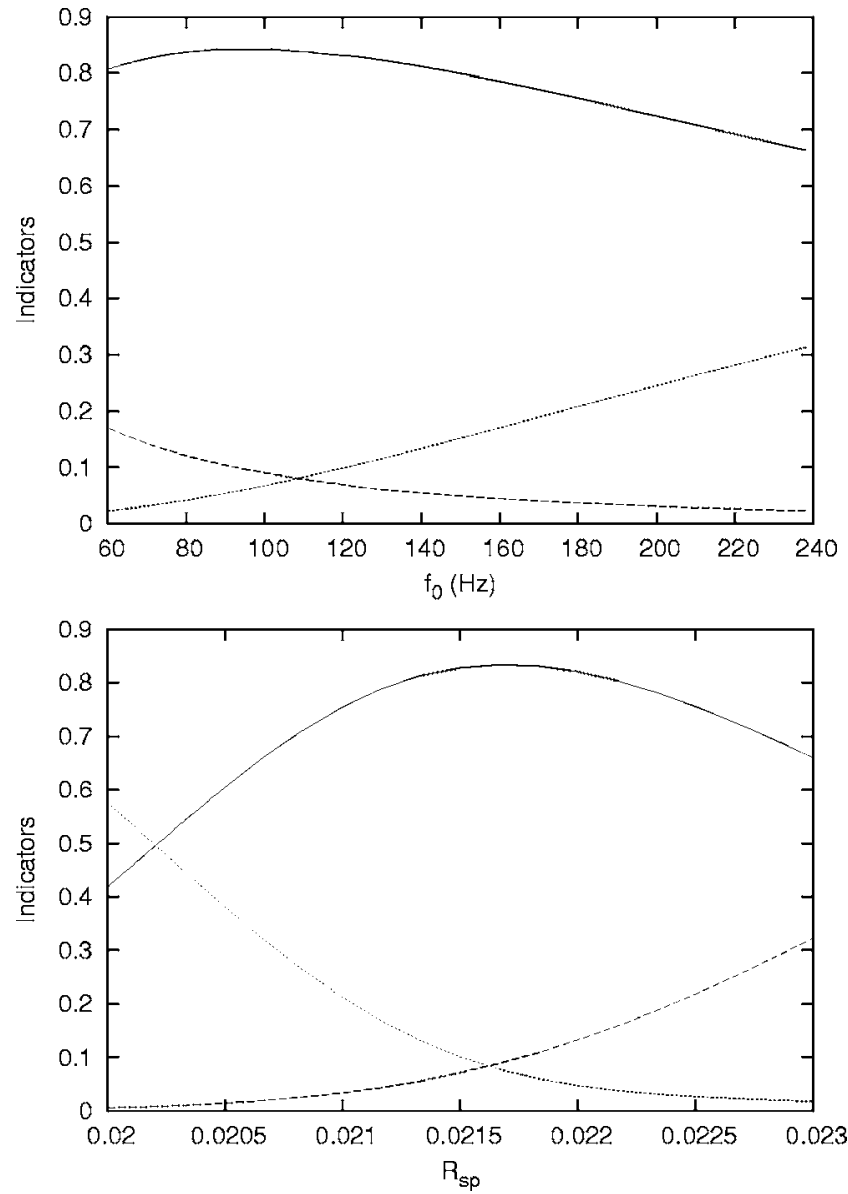

FIG. 11. $I_{T}$ (solid lines), $I_{L}$ (dashed lines), and $I_{H}$ (dotted lines) as a function of the noise strength (left) and as a function of the frequency (right). The indicator $I_{T}$ clearly shows a resonant behavior both as a function of the noise strength and as a function of the frequency. The modulation signal is defined as $J=J_{b}$ $+J_{m} / 2\left(\sin 4 \pi f_{0} t+\sin 6 \pi f_{0} t\right) \quad\left(\delta=3.9, g=3.8, J_{s}=0.64, R_{s p}=0.021\right.$, $\left.J_{b}=0.70, J_{m}=0.12, f_{0}=100 \mathrm{~Hz}\right)$.

In Fig. 11, we plot $I_{T}$ (solid lines), $I_{L}$ (dotted lines), and $I_{H}$ (dashed lines) as a function of the noise strength and as a function of the frequency. The indicator clearly shows a resonant behavior both as a function of the noise strength and as a function of the frequency. In Fig. 12, the ISI distribution is plotted for three different values of the noise strength together with the corresponding spike time distribution functions. For low noise, the spike time distribution is clearly localized. This results in an ISI distribution with peaks at multiples of the period. At high noise, the spike time distribution shows two additional concentrations of probability. The new smaller peak corresponds to spikes which occur at intermediate times. The last very small peak is close to the dominant spike time and hints at a probability that, immediately after a spike has occurred, a second is emitted, resulting in a very small ISI. In all cases, we find a qualitative agreement with the experimental results. In Fig. 9, we have made the comparison between the numerically obtained ISI distribution and the semianalytical results. The two ISI distributions match nicely, showing the validity of the semianalytical theory.
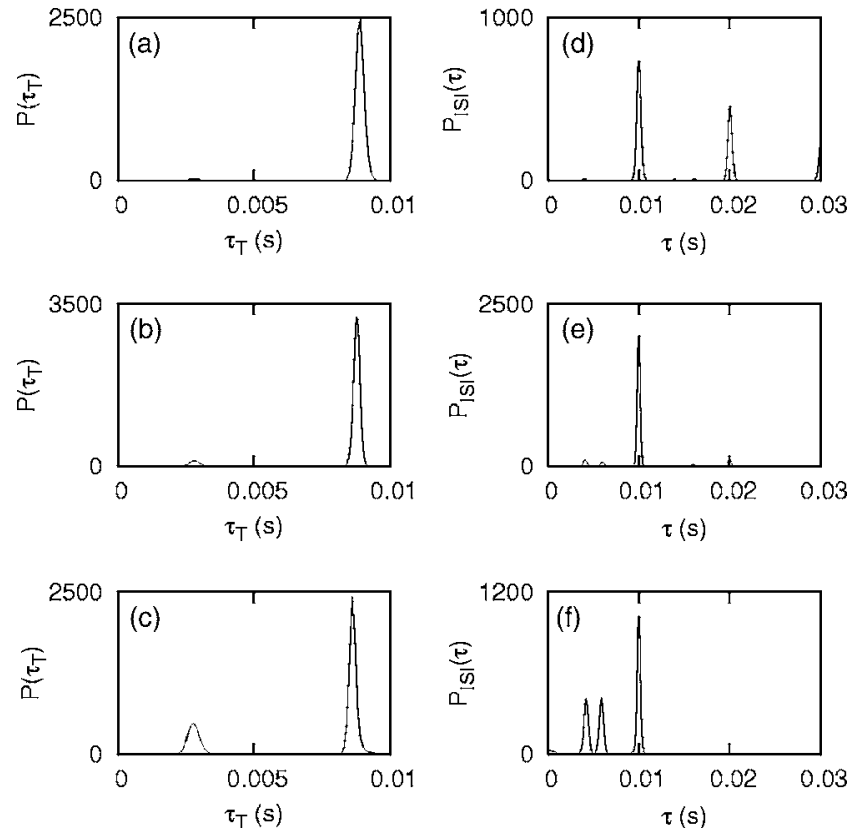

FIG. 12. The semianalytically calculated spike time distributions $P\left(\tau_{T}\right)$ [panels (a), (b), and (c)] and the ISI distributions $P_{I S I}(\tau)$ [panels (d), (e), and (f)] for different noise values. (a)-(d) $R_{s p}$ $=0.019$, (b)-(e) $R_{s p}=0.021$, and (c)-(f) $R_{s p}=0.023$ ( $\delta=3.9, g=3.8$, $\left.J_{s}=0.64, J_{b}=0.70, J_{m}=0.12, f_{0}=100 \mathrm{~Hz}\right)$.

\section{SUMMARY}

We have presented an experimental and theoretical investigation of the ghost resonance phenomenon in a bistable system: namely, a polarization bistable VCSEL. Experimentally, we have shown that ghost resonance indeed occurs in such VCSEL's. The resonance is threewise: in amplitude, in frequency, and in the noise level. GR has been observed for many different combinations of modulation frequency and bias current. On the other hand, we have also shown that in the case of inharmonic signals the resonance can be found at the repetition rates derived by Chialvo et al. [1] from a much simpler model.

Our theoretical description starts from a set of intensity rate equations for a semiconductor laser which takes nonlinear gain effects into account. Using a multiple-time-scale analysis this model has been reduced to a one-dimensional system. From this reduced model an analytical expression for the average residence times was found. We have shown that it is possible to calculate numerically the interspike interval distribution function from the average residence times, effectively reproducing the experimental results in a semianalytical way. Moreover, as this procedure only requires as input the knowledge of the average residence times for different currents, it enables us to predict the ghost resonance point for the VCSEL under study (or any other bistable system). We find a good qualitative agreement between experimental results and theory.

\section{ACKNOWLEDGMENTS}

The authors acknowledge the European RTN network VISTA (Contract No. HPRN-CT-2000-00034) and the COST 


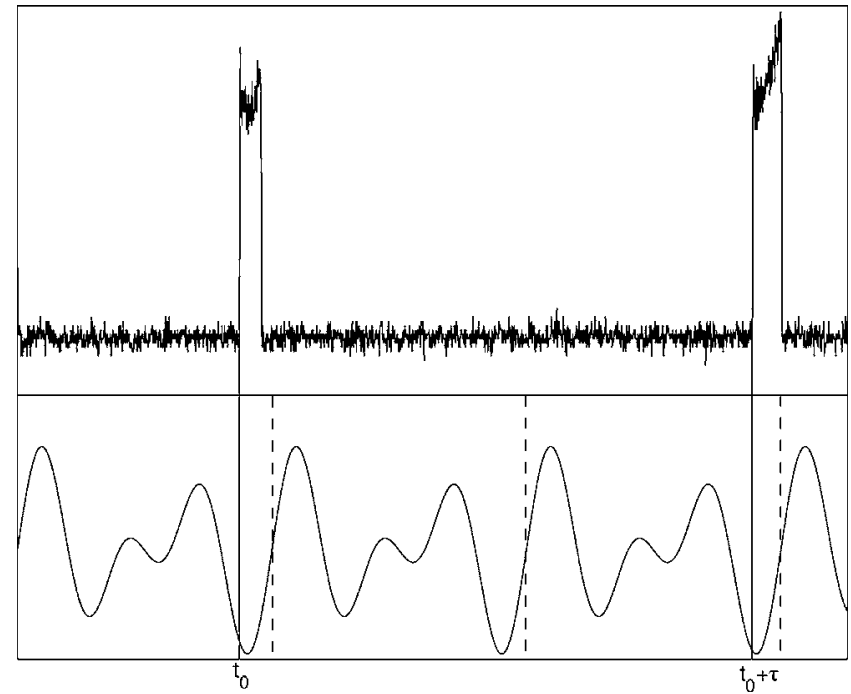

FIG. 13. A typical realization of the spike train for the given modulation signal. The first spike occurs with a spike time $t_{0}$, while the second occurs at a time interval $\tau$ later. The second spike's corresponding spike time is $\bmod _{T}\left(t_{0}+\tau\right)$. To obtain $P\left(\tau_{T}\right)$, we will study the statistics of these spike times.

288 action. J.D., G.V., and G.V.d.S. acknowledge financial and project support from the Research Foundation Flanders (FWO-Vlaanderen). This work was supported on the Belgian side by the Interuniversity Attraction Pole program (IAP V/18), the Concerted Research Action and the Research Council of the Vrije Universiteit Brussel. We thank Professor Rainer Michalzik (U. Ulm) for the devices. C.R.M. acknowledges financial support from the Ministerio de Ciencia y Tecnología (Spain) and FEDER (Project Nos. BFM2000-1108, BFM2001-0341, BFM20001-2159, and BFM2002-04369) and from the EC Project No. IST-2000-29683 OCCULT.

\section{APPENDIX: SPIKE TIME DISTRIBUTIONS AND ISI HISTOGRAMS}

In this appendix, we will show that from the analytical formulation of the residence times in Eq. (16) it is possible to calculate $P\left(\tau_{T}\right)$, and consequently $P_{I S I}(\tau)$, semianalytically.

Before studying the statistics of the time interval between two consecutive rising edges, we take a look at the related statistics of this time interval reduced to within one modulation period. In Fig. 13, we explain the procedure.

Take $t_{0}$ as the time of the rising edge of the first spike. For the realization shown in Fig. 13 the interspike interval equals $\tau$. So the rising edge of the second spike is at $t_{0}+\tau$. At this point, we have skipped one period of the modulation and are at a different point in the next modulation cycle (albeit only slightly different from $t_{0}$ ). This point is equal to $\tau_{T}$ $=\bmod _{T}\left(t_{0}+\tau\right)$, where $\bmod _{T}$ is the modulus function. The distribution of $\tau_{T}$ can then be found to be the conditional ISI distribution folded to one modulation period:

$$
P_{\tau_{T}}\left(\tau_{T} \mid t_{0}\right)=\int_{0}^{\infty} P_{I S I}\left(\tau \mid t_{0}\right) \delta\left(\tau_{T}-\bmod _{T}\left(t_{0}+\tau\right)\right) d \tau .
$$

We will refer to $\tau_{T}$ as the spike time and to $P_{\tau_{T}}\left(\tau_{T} \mid t_{0}\right)$ as the transition probability of the spike times.
Let us now define the distribution of the $k$ th spike time $\chi^{(k)}\left(\tau_{T}\right)$ as the probability that the $k$ th spike in a realization will be at $\tau_{T}$. This probability then transforms from one spike to the next as follows:

$$
\chi^{(k+1)}\left(\tau_{T}\right)=\int_{0}^{T} P_{\tau_{T}}\left(\tau_{T} \mid u\right) \chi^{(k)}(u) d u .
$$

This spike train will approach a stationary Markov process with relative spike time distribution

$$
\chi^{(s)}\left(\tau_{T}\right)=\lim _{k \rightarrow \infty} \chi^{(k+1)}\left(\tau_{T}\right)=\int_{0}^{T} P_{\tau_{T}}\left(\tau_{T} \mid u\right) \chi^{(s)}(u) d u
$$

The stationary spike time distribution $\chi^{(s)}\left(\tau_{T}\right)$ will be the eigenfunction to eigenvalue 1 of the kernel $P_{\tau_{t}}\left(\tau_{T} \mid u\right)$. Due to the fact that $P_{\tau_{t}}\left(\tau_{T} \mid u\right)$ is a conditional probability density function, this eigenfunction should exist [19]. Equation (A3) cannot be solved analytically, but we can solve it numerically by using a binning procedure as described in [20]. The relative spike time is discretized using a number of bins with fixed width $\Delta \tau_{T}$. The relative spike time distribution is then $\chi=\left(\chi_{0}, \chi_{1}, \ldots, \chi_{N-1}\right)^{t r}$ with

$$
\chi_{j}=\int_{j \Delta \tau_{T}}^{(j+1) \Delta \tau_{T}} \chi^{(s)}(u) d u
$$

The relative spike time transition matrix becomes

$$
P_{\tau_{T}, j k}=\int_{j \Delta \tau_{T}}^{(j+1) \Delta \tau_{T}} P_{\tau_{T}}\left(u \mid k \Delta \tau_{T}\right) d u
$$

where $j, k=0,1, \ldots, N-1$. For a given set of parameters, it is now possible to construct numerically Eq. (A5) and then find the corresponding stationary spike time distribution $P\left(\tau_{T}\right)$ $=\chi^{(s)}\left(\tau_{T}\right)$.

To summarize the procedure, using Eq. (16), it is possible to calculate the conditional ISI distributions in Eq. (18). By folding the conditional ISI distribution into one period $T$, we find the transition probability of the spike times [Eq. (A1)] from which we can construct the transition matrix $P_{\tau_{T}, j k}[\mathrm{Eq}$. (A5)]. The eigenvector with eigenvalue 1 of this matrix is the corresponding spike time distribution $P\left(\tau_{T}\right)$. The ISI distribution is then

$$
P_{I S I}(\tau)=\int_{0}^{T} P_{I S I}\left(\tau \mid \tau_{T}\right) P\left(\tau_{T}\right) d \tau_{T}
$$


[1] D. Chialvo, O. Calvo, D. Gonzalez, O. Piro, and G. Savino, Phys. Rev. E 65, 050902(R) (2002).

[2] D. Chialvo, Chaos 13, 1226 (2003).

[3] J. Buldú, D. Chialvo, C. R. Mirasso, C. Torrent, and J. GarcíaOjalvo, Europhys. Lett. 64, 178 (2003).

[4] O. Calvo and D. Chialvo (unpublished).

[5] G. Giacomelli, F. Marin, and I. Rabbiosi, Phys. Rev. Lett. 82, 675 (1999).

[6] S. Barbay, G. Giacomelli, and F. Marin, Phys. Rev. E 61, 157 (2000).

[7] B. Nagler, M. Peeters, I. Veretennicoff, and J. Danckaert, Phys. Rev. E 67, 056112 (2003).

[8] H. Unold, S. Mahmoud, R. Jaeger, M. Grabherr, R. Michalzik, and K. Ebeling, IEEE J. Sel. Top. Quantum Electron. 7, 386 (2001).

[9] F. Marchesoni et al., Phys. Rev. E 58, 7079 (1998).

[10] F. Marchesoni et al., Phys. Rev. E 59, 3958 (1999).
[11] L. Gammaitoni, F. Marchesoni, and S. Santucci, Phys. Rev. Lett. 74, 1052 (1995).

[12] L. Gammaitoni, P. Hänggi, P. Jung, and F. Marchesoni, Rev. Mod. Phys. 70, 223 (1998).

[13] M. Choi, R. Fox, and P. Jung, Phys. Rev. E 57, 6335 (1998).

[14] R. Löfstedt and S. Coppersmith, Phys. Rev. Lett. 72, 1947 (1994).

[15] R. Löfstedt and S. Coppersmith, Phys. Rev. E 49, 4821 (1994).

[16] F. Marchesoni et al., Phys. Rev. E 62, 146 (2000).

[17] J. Danckaert, B. Nagler, J. Albert, K. Panajotov, I. Veretennicoff, and T. Erneux, Opt. Commun. 201, 129 (2002).

[18] B. Nagler et al., Phys. Rev. A 68, 013813 (2003).

[19] C. Baker, The Numerical Treatment of Integral Equations (Clarendon, Oxford, 1977).

[20] H. Plesser and T. Geisel, Phys. Rev. E 59, 7008 (1999). 